Erschienen in: Blühdorn, Hardarik/Breindl, Eva/Waßner, Ulrich Hermann (Hrsg.):

\title{
Relationsbedeutung und Konnektorbedeutung: Additivität, Adversativität und Konzessivität
}

\section{Zielsetzung}

So vielfältig die Ausdrucksmöglichkeiten für Kontrast im Deutschen und in anderen Sprachen sind (s. die eindrucksvolle Liste in Rudolph 1996: 4f.), so unklar ist nach wie vor, auf welcher Basis die Kontrastrelation sinnvoll von anderen Relationen abzugrenzen und intern zu strukturieren ist. Seit je war dabei die Abgrenzung einer allgemeineren Relation Kontrast (auch Adversativität genannt) von einer vermeintlich spezielleren Konzessivität ein Brennpunkt. Untrennbar damit verbunden ist das Problem einer adäquaten und möglichst ohne Polysemieannahmen auskommenden Bedeutungsbeschreibung für aber, das einerseits als prototypischer adversativer Konnektor angesehen wird, gleichzeitig aber die durch monoseme Konnektoren wie obwohl, obgleich, trotzdem ausgedrückte Konzessivität mit abdecken kann.

Der vorliegende Beitrag ${ }^{1}$ versucht, Überlappungen und Abgrenzungen von Relationsbedeutungen - verstanden als gemeinsamer Bedeutungsanteil der Ausdrücke für diese Relation - und den Bedeutungen einzelner Konnektoren im Kontrastfeld aufzuzeigen. Evidenzen für Verortung und Strukturierung der Kontrastrelation werden dabei aus zwei Beschreibungsdimensionen gewonnen: Zum einen lässt sich auf der Basis formaler Parameter, wie sie in der Grammatikalisierungsforschung verwendet werden (z.B. Lehmann 1995a und b), eine skalare Anordnung von Subtypen bzw. Teilfeldern festmachen und Kontrast auch gegenüber der bislang weniger beachteten Relation Additivität verorten. Zum anderen können, gestützt durch systematischen Austausch mit semantisch spezifischeren Kontrastkonnektoren (allerdings, dafür, dennoch, hingegen, obwohl, während etc.), verschiedene Verwendungsweisen von aber isoliert und Differenzparameter ermittelt werden, die sich als unterschiedliche kontextspezifische Ausprägungen einer Grundbedeutung lesen lassen. Damit können Umschlagpunkte in der Kodierung von Additivität, Adversativität und Konzessivität ermittelt und die Relationsbedeutungen besser herauspräpariert werden.

1 Für hilfreiche Kritik und Kommentare danke ich Renate Pasch und Gisela Zifonun. 


\section{Der Relationsausschnitt Additivität, Adversativität, Konzessivität}

Der Ausschnitt Additivität, Adversativität, Konzessivität aus dem Gesamtinventar semantischer Relationen bedarf einer kurzen Begründung. Allen dreien liegt eine logische Konjunktion zugrunde; das grenzt sie von konditionalen, die wiederum etwas gemeinsam haben mit konzessiven -, nicht aber von kausalen und vielen temporalen Konnektoren ab. Adversativität und Konzessivität werden hier zunächst verstanden als voneinander distinkte Relationen (was nicht heißen muss, dass es bei den einzelnen Sprachzeichen keine Überschneidung gibt), die zusammen mit anderen Relationen Teilbereiche einer übergeordneten unspezifischeren Relation „Kontrast“ sind. Als „Wesen“ des Kontrasts, d.h. als gemeinsames Merkmal aller Kontrastrelationen und gleichzeitig Differenzparameter gegenüber der Additivität sei ein konträres Verhältnis des internen Arguments des Konnektors zu etwas, was in irgend einer Weise aus dessen externem Argument ableitbar ist, angenommen. In der Bedeutungsstruktur der Relation muss also eine Negation vorhanden sein, wobei zunächst außer Acht bleiben soll, auf welcher Ebene - propositional, präsuppositional und zwischen welchen Bestandteilen der Argumente diese Negation genau anzusiedeln ist. Eine Kontrastinterpretation unterliegt damit schärferen Bedingungen im Verhältnis der Relata zueinander als der bloßen Distinktheitsbedingung, die Eingangsbedingung für Satzverknüpfungsrelationen generell ist (Lang 1991).

\subsection{Additiv und adversativ: und - aber}

Für das Verhältnis von Additivität und Adversativität, als deren prototypische Repräsentanten und und aber gelten, wird in der Regel unter Hinweis auf die logische Konjunktion ein Inklusionsverhältnis postuliert. So etwa in der Grundzüge-Grammatik: „Das adversative Verhältnis gilt als besondere Ausprägung der ,Konjunktion““ [ = logische Konjunktion, EB] (1981: 701) oder bei Lang (1975: 102): „Durch ein aber wird der durch und gesetzte Zusammenhang allemal mitrepräsentiert." Minimalpaare, in denen und und aber austauschbar scheinen, werden dafür als Beleg herangezogen.

Die Eltern sprechen türkisch und die Kinder sprechen deutsch. Die Eltern sprechen türkisch, aber die Kinder sprechen deutsch.

In beiden Fällen wird ein Vergleich der in den Konnekten ausgedrückten Sachverhalte vor dem Hintergrund einer „gemeinsamen Einordnungsinstanz“ GEI (Lang 1977) etabliert. Genauer gesagt werden die beiden kontrastiven Satztopiks Eltern und Kinder, die komplementäre Instanzen einer übergeord- 
neten Kategorie ,Familie“ sind, hinsichtlich einer bestimmten Charakteristik, nämlich ihres Sprachgebrauchs, verglichen. Auf den ersten Blick scheinen und- und aber-Verknüpfungen dieses Typs äquivalent. Bei der aber-Verknüpfung wird der Vergleich jedoch als kontrastierend und die jeweils alternativen Belegungen als ausgeschlossen präsentiert: Es ist nicht der Fall, dass die Eltern deutsch sprechen, und es ist nicht der Fall, dass die Kinder türkisch sprechen. Unter Berücksichtigung der Kontexteinbettung sind und und aber, wie Brauße (1998) gezeigt hat, nicht mehr ohne weiteres vertauschbar.

(1a) [A: Wie wird in der Familie gesprochen? - B:] Die Eltern sprechen türkisch undlaber die Kinder sprechen deutsch.

(2a) [A: In der Familie wird wohl türkisch gesprochen? - B: Nein,] Die Eltern sprechen türkisch, aber/*und die Kinder sprechen deutsch.

Die Vorstellung eines Inklusionsverhältnisses ist vor allem mit (2a) nicht verträglich, wo das vermeintlich allgemeinere und nicht das spezifischere aber ersetzen kann. Und und aber stehen vielmehr in einem polaren Verhältnis: Sie unterscheiden sich in einem relativ allgemeinen Bedeutungsmerkmal $<$ BüNDELUNG $>$, das bei und positiv, bei aber negativ spezifiziert ist (so Brauße 1998). Additivität, <+BÜNDELUNG>, kann mit Lang (1991) ausbuchstabiert werden zu „fasse die Konnektbedeutungen als Instanzen einer gemeinsamen Einordnungsinstanz zusammen“. Mit dem Bündelungs-Merkmal geht die Bedeutung von und auch über die Bedeutung der aussagenlogischen Konjunktion hinaus, die ja außer bei einer Reihe anderer Relationen auch bei asyndetischen Verknüpfungen gegeben ist. Insofern dem positiv spezifizierten Merkmal von und ein negatives bei aber gegenübersteht, - aufgrund des Kontrastverhältnisses sind die Konnektbedeutungen gerade nicht „bündelbar“ - ist die aberRelation semantisch markierter und komplexer. ${ }^{2}$

\subsection{Adversativ und konzessiv: aber-obwohl}

Das Verhältnis von Adversativität und Konzessivität ist in nahezu allen Beschreibungen von aber (resp. but, mais, maar) zentral. In der Tradition von Lakoff (1971) werden dafür zwei Bedeutungen angenommen: ein „semanticopposition-but" und ein „denial-of-expectation-but".

(2) Die Eltern sprechen türkisch, aber die Kinder sprechen deutsch.

(3) Die Eltern sprechen türkisch, aber sie lesen deutsche Zeitungen.

2 Die Ersetzbarkeit von aber durch und in bestimmten Kontexten erklärt sich dann als Fall von Neutralisierungskontext, in dem der unmarkierte Pol verwendet wird, vergleichbar der Neutralisierung bei polaren Adjektiven wie groß - klein, schwer - leicht in Ausdrücken wie $20 \mathrm{~cm}$ groß, $100 \mathrm{~g}$ schwer. 
(2) steht für den semantic-opposition-Typ bzw., treffender, „kontrastiven Vergleich“ der Satztopiks (Übersetzung von Foolens (1991) ,,contrastive comparison“). (3) ist ein Fall von „denial-of-expectation“, i.e. „Behauptung entgegen andersliegender Erwartung“ (Lang 1977) oder „konzessives“ aber. Für das konzessive aber wird in aller Regel eine Bedeutungsbeschreibung über ein Konzept des „Widerspruchs gegen die Normalerwartung“ getroffen, deren Basis ein Konglomerat aus kollektiv geteiltem Weltwissen, kulturellen Bewusstseinsinhalten und Bewertungsmustern ist. Der kontrastive Vergleich wurde vielfach als Spezialfall des konzessiven angesehen. Andererseits wurde wiederum Konzessivität als Spezialfall einer allgemeineren „Adversativität“ beschrieben (vgl. beispielsweise Di Meola in diesem Band, der Adversativität etwa so verwendet, wie hier Kontrast verwendet wird), oder es wird bewusst auf eine Unterscheidung verzichtet.

Festzuhalten ist zunächst, dass (2) und (3) im Deutschen unterschiedliche Substitutionsmengen von Konnektoren haben.

(2b) Während die Eltern türkisch sprechen, sprechen die Kinder deutsch. (hingegen, dagegen, demgegenüber, indes)

(2c) $\quad \neq$ Obwohl die Eltern türkisch sprechen, sprechen die Kinder deutsch.

(3b) Obwohl die Eltern türkisch sprechen, lesen sie deutsche Zeitungen. (obgleich, trotzdem, dennoch)

(3c) $\quad \neq$ Während die Eltern türkisch sprechen, lesen sie deutsche Zeitungen.

Die Bedeutung von aber ist also unspezifischer als die rein konzessive von obwohl, obgleich, trotzdem etc. und als die von hingegen, dagegen, während etc., die den kontrastiven Vergleich markieren. Die Konnektoren und, aber, obwohl sind - als prototypische Vertreter der Relationen „Additivität“, „Adversativität“ und „Konzessivität“ - auf einer Skala zunehmender semantischer Komplexität und abnehmender Vagheit angeordnet. Dabei hat und, wie Posner (1979) gezeigt hat, von allen Konnektoren den breitesten Interpretationsspielraum.

(a) $\rightarrow$ kontrastiver Vergleich (,hingegen'): Jungs sind trotzig, und Mädchen sind fügsam.

(b) $\rightarrow$ konzessiv (,trotzdem'): Sie ist erst 17, und hat zwei Kinder!

(c) $\rightarrow$ temporal-sukzessiv (,darauf'): Er kam, sah und siegte.

(d) $\rightarrow$ konditional (,dann'): Setze auf die 13 und du gewinnst.

(e) $\rightarrow$ konklusiv (,folglich'): Mia fiel hin und verstauchte sich den Knöchel.

(f) $\rightarrow$ instrumental (,dadurch'): Mia haut auf den Stein und zertrümmert ihn.

Diese und-Interpretationen sind freilich nicht völlig willkürlich: Die „symmetrischen" Verwendungen (a) und (b) sind klare Fälle von <BüNDELUNG $>$, in 
denen ein Kontrast sich via Inferenz aus den Konnektbedeutungen ergibt. In den übrigen Fällen wird das Bündelungskonzept ikonisch interpretiert: in (c) als chronologische Ereignisfolge von Sachverhalten, in (d) bis (f) als logische Ereignisfolge von Prämisse und Folge. Solche ikonischen Interpretationen existieren in allen Sprachen für den je prototypischen „Bündelungskonnektor“, und sie greifen auch bei parataktischen Satzfolgen, sind also nicht lexikalische Spezifika von und (vgl. Sweetser 1990: 87) ${ }^{3}$.

$A b e r$ lässt wie gezeigt unter anderem konzessive Interpretation zu und $o b$ wohl ist die „Endstation für jede Interpretationserweiterung“ (König 1991: 632). In einem nächsten Schritt ist zu prüfen, ob mit dieser lexikalisch-semantischen Komplexitätszunahme auch formal-syntaktische Eigenschaften korreliert sind.

\section{Formale Eigenschaften der Konnektoren: und - aber-obwohl}

\subsection{Morphonologisches Gewicht}

Und, aber, obwohl sind bezüglich ihres morphonologischen Gewichts skalar geordnet. Und ist ein Simplex, aber eine synchron verdunkelte Komparativbildung zu *ap (,weg', ,fort', ,ab') mit der Grundbedeutung ,weiter entfernt', ,später', obwohl und alle anderen konzessiven Konnektoren sind transparente Zusammenrückungen bzw. Komposita aus Syntagmen (wenngleich, obzwar) oder deverbalen (dessen ungeachtet) und denominalen (trotzdem) Bestandteilen. Dass die semantische Komplexität der Konzessivrelation sich in der morphologischen Komplexität konzessiver Konnektoren „spiegle“ führt auch Di Meola (in diesem Band) an. Unter den additiven Konnektoren finden sich mit wie (Männer wie Frauen) und auch weitere Simplizia, unter den adversativen ist nur doch Simplex, alle anderen sind morphologisch komplex, meist von der Form des Pronominaladverbs (hingegen, demgegenüber, andererseits) mit räumlich-lokaler Etymologie.

\subsection{Phylogenese und Ontogenese}

Auch Diachronie und Spracherwerbsverlauf bestätigen die Anordnung. Konzessive Bildungen sind eher jungen Datums (s. König 1985) und werden spät

3 Die Zugänglichkeit von und für ikonische Interpretationen liegt sicherlich auch daran, dass die Bündelung von Sachverhalten unter einer gemeinsamen Einordnungsinstanz als mentale Operation nicht allzu weit über die Herstellung diskurspragmatischer Kohärenz hinausgeht, und dadurch zu interpretatorischer Anreicherung geradezu einlädt. 
gelernt (Di Meola 1997: 10). Und erscheint im Spracherwerb vor aber, das relativ früh und als erster Kontrastmarker erworben wird. Diese Reihenfolge ist auch für den Zweitspracherwerb belegt: Unter den zehn frequentesten Konjunktionen in Lernervarietäten bei Birkner et al. (1995) erscheint und an erster Stelle mit deutlichem Abstand vor aber, Konzessiva kommen nicht vor.

\subsection{Erweiterungsmöglichkeiten der Klassen}

Keine der drei Klassen ist völlig geschlossen, da Erweiterungen durch Adverbbildungen möglich sind; sieht man von diesen $a b$, präsentiert sich die Klasse der additiven Konnektoren aber am „geschlossensten“. Subjunktoren jüngeren Datums gibt es nur unter den adversativen und konzessiven.

additiv: Zugänge vor allem aus lokalen Adverbien: ferner, zudem, darüber hinaus, überdies, außerdem, ältere Übertritte bei Konjunktoren aus komparativen Adverbien: wie, sowie, sowohl (...) als auch

adversativ: Zugänge vor allem aus lokalen und temporalen Adverbien: demgegenüber, dementgegen, da(hin)gegen, einerseits (...) ander(er)seits, zum einen (...) zum anderen, indes(sen); teilweise Weiterentwicklung zu Subjunktoren: wo(hin)gegen, wobei, wogegen, indessen

konzessiv: Zugänge bei Adverbien: (nichtsdestotrotz, nichtsdestominder, des(sen)ungeachtet, ungeachtet dessen); relativ junge Subjunktoren: obgleich, obzwar, obschon, wiewohl, wenn auch, wenngleich, trotzdem

\section{Syntagmatische Eigenschaften der Konnektoren}

\subsection{Verkettungsmodus: Grammatizitätszunahme - Pragmatizitätsabnahme}

Additive Konnektoren sind Konjunktoren und Adverbien, verknüpfen also koordinativ oder über Adverbintegration. Adversative Konnektoren verknüpfen fast ausschließlich über Adverbintegration; lediglich aber hat mit der intonatorisch nicht abgegrenzten Position zwischen den Konnekten auch gewisse Züge von Konjunktoren, und mit während, wo(hin)gegen und dem stilistisch markierten indessen liegen die einzigen adversativen Subjunktoren des Deutschen vor. Konzessive Konnektoren sind überwiegend Subjunktoren und Adverbien, verknüpfen also subordinativ und via Adverbintegration. 


\begin{tabular}{|c|c|c|}
\hline $\begin{array}{l}\text { und, sowie, } \\
\text { sowohl als auch }\end{array}$ & $\begin{array}{l}\text { additiv } \\
\text { ferner, zudem, überdies, } \\
\text { daneben, darüber hinau }\end{array}$ & \\
\hline \multirow[t]{2}{*}{ aber } & \begin{tabular}{l}
\multicolumn{1}{c}{ adversativ } \\
da(hin)gegen, nur, \\
demgegenüber, allein, \\
jedoch, allerdings
\end{tabular} & $\begin{array}{l}\text { während, } \\
\text { wo(hin)gegen, } \\
\text { indessen }\end{array}$ \\
\hline & $\begin{array}{l}\text { konzess } \\
\text { trotzdem, } \\
\text { dennoch, } \\
\text { nichtsdestominder }\end{array}$ & $\begin{array}{l}\mathrm{v} \\
\text { obwohl, obschon, } \\
\text { wenngleich, } \\
\text { wenn auch }\end{array}$ \\
\hline
\end{tabular}

Die drei Komplexbildungsverfahren sind hinsichtlich der in Pasch et al. (2003) klassifikatorisch verwendeten und näher erläuterten Merkmale <INTEGRATION>, d.h. positionelle Integration des Konnektors in das interne Konnekt, $<$ EINBETTUNG>, d.h. Möglichkeit der Stellung des internen Konnekts im Vorfeld des externen und $<$ PHRASENBILDUNG $>$, d.h. Bildung einer Phrase mit dem Konnektor als Kopf und seinem internen Konnekt als Komplement, wie nachstehend aufzuschlüsseln.

\begin{tabular}{|l|l|l|}
\hline \multicolumn{1}{|c|}{ Koordination } & \multicolumn{1}{c|}{ Adverbintegration } & \multicolumn{1}{c|}{ Subordination } \\
\hline Verkettung koordinativ & Verkettung parataktisch & Verkettung subordinativ \\
\hline$<-$ INTEGRATION $>$ & $<+$ INTEGRATION $>$ & $<-$ INTEGRATION $>$ \\
\hline$<-$ EINBETTUNG $>$ & $<-$ EINBETTUNG $>$ & $<+$ EINBETTUNG $>$ \\
\hline$<-$ PHRASENBILDUNG $>$ & $<-$ PHRASENBILDUNG $>$ & $<+$ PHRASENBILDUNG $>$ \\
\hline
\end{tabular}

Es zeigt sich das typische Bild einer Anordnung vom ,pragmatischen Verkettungsmodus“ zum „syntaktischen“ oder „grammatischen“, die durch den bekanntlich vom pragmatischen zum grammatischen schreitenden Spracherwerbsverlauf bestätigt wird. ${ }^{4}$

4 „Pragmatischer" sind Koordination und Adverbintegration auch deshalb, weil hier in der Regel im internen Konnekt ein Vorfeld vorhanden ist, dessen Besetzung eher diskurspragmatisch als strukturell motiviert ist. Bei Subordination. existiert dagegen nur ein Mittelfeld, dessen Besetzung stärker über strukturelle Eigenschaften und semantische Skopuseigenschaften der Konstituenten als diskurspragmatisch bestimmt ist. 


\subsection{Zunehmende Restringiertheit der Konnektformate}

Koordination erlaubt Verknüpfung von konstituentenkategoriell beliebigem, hinsichtlich der syntaktischen Funktion gleichrangigem Material: Äußerungen aller Satzmodustypen, Sätze aller Verbstellungstypen, Phrasen, Wörter, Morpheme. Die Möglichkeiten der Koordinationsreduktion sind ausgeprägt und Verknüpfungen von mehr als zwei Konnekten sind möglich.

Adverbkonnektoren erlauben Verknüpfung von Aussagesätzen; sie sind nur eingeschränkt in andere Satzmodus- bzw. Verbstellungstypen integrierbar. Adverbkonnektoren haben immer nur zwei Konnekte: ein Trägerkonnekt als internes Argument und ein in der Linearität diesem voraufgehendes Bezugskonnekt als externes Argument. Die Möglichkeiten der sprachlich gestützten Ellipsenbildung sind im Vergleich zu Koordinationsellipsen eingeschränkt (s. Pasch et al. 2003: 549f.). Beim syntaktisch ,zwittrigen“ aber sind koordinativ gestützte Weglassungen noch eher möglich als bei anderen Adverbkonnektoren (vgl. (4b) gegen (4c)), und generell sind Weglassungen im externen Konnekt von Adverbkonnektoren nicht in dem Maße möglich wie Weglassungen im externen Konnekt von Konjunktoren: Rückwärtsellipsen sind jedenfalls ausgeschlossen (vgl. (4g) gegen (4h)).

(4a) Hans ist klein und Fritz groß.

(4b) Hans ist klein aber Fritz groß.

(4c) *Hans ist klein, dagegen/indes/demgegenüiber/trotzdem Fritz groß.

(4d) *Hans ist klein, während Fritz groß.

(4e) Hans ist groß und Fritz.

(4f) Hans ist groß, Fritz auch.

(4g) Hans und Fritz sind groß.

(4h) *Hans, Fritz auch sind groß.

Subordinierende Konnektoren erzwingen Verbletztstellung im internen Konnekt, sind also im Unterschied zu Konjunktoren und Adverbien regierende Ausdrücke; die Ellipsenbildung ist beschränkt und spezifisch lexikalisch (s. Pasch et al. 2003: 361ff.). Das gilt auch für den eher dem „pragmatischen“ Pol zuzurechnenden Konstruktionstyp der Desintegration (Wenn es auch stürmt und schneit, wir fahren nach Speyer). Zumindest für die konzessiven Subjunktoren gilt dabei, wie Di Meola (in diesem Band) zeigt, dass gerade die hochgradig grammatikalisierten (obwohl, obschon, obzwar) deutlich seltener in Desintegrationskonstruktionen verwendet werden als die niedriggradig grammatikalisierten mehrteiligen. 


\subsection{Zunehmende Stellungsfreiheit der Konnekte}

Gegenläufig zur Restringiertheit der Konnektformate unterliegt die Abfolge der Konnekte abnehmenden Beschränkungen. Konjunktoren und Adverbkonnektoren erlauben nur die Reihenfolge externes Konnekt vor internem, Subjunktoren auch die umgekehrte. Damit bieten sie eine größere Variationsbreite für die Informationsstruktur. ${ }^{5}$

Fazit: Die zunehmende semantische Komplexität der Relationsbedeutungen von Additivität, Adversativität und Konzessivität korreliert mit einer Komplexitätszunahme in den formalen und syntagmatischen Eigenschaften der Konnektoren. Gegenläufig dazu nimmt der Interpretationsspielraum eines Konnektors mit seiner formalen und syntagmatischen Komplexität ab. ${ }^{6}$ Auf einer Grammatikalisierungsskala mit den Polen „grammatisch“ und „lexikalisch" bzw. Synsemantika und Autosemantika, wie in Lehmann (1995a) skizziert, wären die additiven Konnektoren näher am grammatischen, die konzessiven näher am lexikalischen Pol anzusiedeln. Bezogen auf die Verkettungsverfahren zeigt sich ein anderes Bild: auf einer Skala ,pragmatisch - grammatisch" erweisen sich additive, adversative und konzessive Konnektoren als zunehmend grammatisch und abnehmend pragmatisch. M.a.W.: die syntaktische Verknüpfung zwischen den Konnekten ist umso enger, je komplexer die Konnektorbedeutung und damit die semantische Relation zwischen den Konnekten ist. In einem nächsten Schritt ist nun zu prüfen, inwieweit semantische Parameter, nämlich das Verhältnis der Konnektbedeutungen zueinander und zum Kontext mit dieser Anordnung korrespondieren. Diese Parameter sind gleichzeitig relevante Dimensionen bei der semantischen Beschreibung von Konnektoren. Auf eine weitere, an dieser Stelle vielleicht erwartete beschreibungsrelevante Dimension, die Verknüpfungsdomänen in der Tradition von Sweetser (1990), gehe ich im Zusammenhang mit Verwendungstypen von aber ein.

5 Gegenüber der syntaktischen Subklassifikation von Konnektoren im HdK ist die hier benutzte etwas gröber. Die dort ermittelten „Übergangsklassen“ zwischen Koordination und Subordination, Verbzweitsatz-Einbetter und Postponierer, werden hier nicht eigens berücksichtigt, sie zeigen gegenüber Subjunktoren Einschränkungen in der Fokus-Hintergrund-Gliederung insofern als immer beide Konnekte fokal sein müssen (Postponierer) oder zumindest bevorzugt fokal sind (Verbzweitsatz-Einbetter).

6 Eine vergleichbare Skala von schwacher semantischer zu starker semantischer Relation haben König/Van der Auwera (1988) für den Relationsausschnitt Irrelevanzkonditionale - Konditionale - Konzessive aufgezeigt. Kortmann (1998) weist die Gültigkeit der „Inverse Relation Hypothesis“, wonach formale Komplexität und semantische wie syntaktische Polyfunktionalität sich umgekehrt proportional zueinander verhalten (ebd. 478), für ein breites Sprachenspektrum nach. 


\section{Dimensionen der semantischen Beschreibung von Kontrast- Konnektoren}

\subsection{Verhältnis der Konnektbedeutungen zueinander}

Bei additiven Verknüpfungen und beim kontrastiven Vergleich sind die Bedeutungen der Konnekte unabhängig voneinander und miteinander verträglich (vgl. Lang 1991: 606f.). Bei konzessiven Verknüpfungen sind zwar die Konnektbedeutungen selbst ebenfalls unabhängig, der Konnektor induziert jedoch auf der präsuppositionalen Ebene einen implikativen Zusammenhang zwischen den Konnektbedeutungen: wenn $\mathrm{p}$, dann normalerweise nicht q. Dieser ergibt sich nicht unmittelbar aus den Konnektbedeutungen selbst, sondern unter Rekurs auf kollektiv verfügbare Schlussschemata des Alltagswissens und wird durch die Konnektorbedeutung erzwungen. Scheinbar absurde Beispiele für Konzessivität lösen - Sinnhaftigkeit vorausgesetzt - beim Hörer Raisonnements aus, einen situativ angemessenen Zusammenhang zwischen Folgerungen aus den Konnektbedeutungen herzustellen oder wirken, wo dies nicht gelingt, so befremdlich wie (5).

Fahren Sie vorsichtig und kommen Sie trotzdem gut an. (Verkehrsmeldung in SWR 1, 3.5.03)

\subsection{Symmetrie vs. Asymmetrie der Relation}

Symmetrie sei hier verstanden als Möglichkeit, die lautlich realisierten Konnekte „über Kreuz" den semantischen Rollen zuzuordnen, die den Konnekten als relationale Bedeutungen durch die Konnektorbedeutungen zugewiesen werden, nicht zu verwechseln mit der in 3.3 diskutierten bloßen topologischen Vertauschung der Konnektausdrücke. Die Übertragung des Konzepts der semantischen Rollen vom Verbalbereich auf den der Konnektoren setzt voraus, dass man das Verhältnis von Konnektor zu Konnekten als Funktor-ArgumentStruktur analysiert, die bei den subordinierenden und einbettenden Konnektoren analog zum Verbalbereich auch syntaktisch als Kopf-Komplement-Beziehung realisiert ist. Für die Rollen der Argumente von Konnektoren gibt es in noch weit geringerem Maße als für die der Verbargumente ein konsensfähiges Inventar; es findet sich eine Vielzahl von Begriffen wie „Grund“, „Folge“, „Ziel“", „Bedingung“, „,unwirksamer Gegengrund“ etc., die aber durchweg nicht terminologisiert und definiert sind. ${ }^{7}$

7 Die Entwicklung eines Rolleninventars für Konnektoren, mit dessen Hilfe systematische Bezüge zwischen den Konnektoren präzise abgebildet werden können (z.B. die diathesenähnliche Beziehung zwischen Subjunktoren und Adverbkonnektoren wie obwohl - trotzdem; 
Bei additiven Konnektoren sind diese Rollen identisch und kaum spezifischer zu fassen als „Instanz einer Bündelung unter einem gemeinsamen Einordnungsaspekt“. Die Verteilung nach „Basis“ und „Erweiterung“ ist - zumindest bei Konjunktoren - eine Frage pragmatischer Implikaturen und ikonischer Interpretation unter bestimmten Kontextbedingungen und die Bedeutung von

(6a) Anna hat geheiratet und ein Kind bekommen. und

(6b) Anna hat ein Kind bekommen und geheiratet.

ist abgesehen davon gleich, die Implikatur ist aufhebbar und eine Unterscheidung zwischen „symmetrischem“ und ,asymmetrischem“ und in Fällen wie (6b) oder den in 1.2 diskutierten ist nicht sinnvoll.

(6a') Anna hat geheiratet und ein Kind bekommen, aber damit will ich nicht sagen, dass sie das Kind erst nach der Heirat bekommen hat.

Bei adversativen Konnektoren ist der Befund etwas komplizierter. Beim kontrastiven Vergleich scheint Symmetrie gegeben (vgl. a. Lakoff 1971):

Während Fritz groß ist, ist Hans klein. (aber, dagegen, hingegen) Während Hans klein ist, ist Fritz groß.

Sobald aber Bewertungen eine Rolle spielen, ändert eine Vertauschung die „argumentative Orientierung“ (Anscombre/Ducrot 1977). Adversative Konnektoren, die bewertete Konnekte verknüpfen können, sind nach zwei Dimensionen spezifiziert (s. Breindl im Druck b): zum einen nach dem Wert des internen Arguments, das diesbezüglich positiv (dafür, immerhin), negativ (nur) oder indifferent (aber, allerdings) sein kann; zum anderen nach der argumentativen Gewichtung: Aber ist - in der Verwendung als „Bewertungsgegensatzaber" - asymmetrisch und weist seinem internen Konnekt stärkeres argumentatives Gewicht zu. ${ }^{8}$ Das bestimmt auch mögliche Folgerungen aus der gesamten Verknüpfung. In (8a) ist deshalb die kontrastive Verknüpfung eine kon-

wenn (nicht) - andernfalls oder Verwandtschaften zwischen Klassen), ist ein echtes Forschungsdesiderat. Ein Inventar definierter Rollen ist derzeit am ehesten noch in den Kohärenzrelationen der Rhetorical Structure Theory erkennbar (s. Stede in diesem Band).

8 Die argumentative Asymmetrie von aber ist in der Literatur nicht unumstritten. Sie findet sich in der weit verbreiteten aber-Explikation von König (1991), der sich auf Anscombre/Ducrot (1977) beruft; nicht aber z.B. bei Lang $(1991,1977)$ oder Rudolph (1996). Da sie in nicht bewertenden Kontexten nicht wirksam wird, und auch nicht zweifelsfrei nachweisbar ist, dass andere Kontrastkonnektoren konträre Bewertungen so verknüpfen können, dass das erste (externe) und nicht das zweite (interne) Argument stärkeres argumentatives Gewicht hat, ist sie vermutlich kein lexikalischer Bestandteil von aber, sondern kommt generell bei Bewertungen ins Spiel und ist der unmarkierten Fokus-Hintergrund-Gliederung mit der stärkeren Gewichtung am Ende geschuldet, ist also ein kontextuell-diskursiver Faktor. 
gruente Exemplifizierung der im voraufgehenden Kontext verbalisierten positiven Wertung langsam geht es voran, während die Konnektvertauschung in (8b) zu einer gewissen Inkohärenz führt.

Langsam geht es auch auf den anderen Baustellen voran. Etwa dort, wo nach osteuropäischem Vorbild neue Wohnblocks aus Betonfertigteilen entstehen - [...] keine architektonischen Kleinode, aber mit Stromanschluß, fließendem Wasser und Zentralheizung.

(taz, 22.12.1986, S. 8)

?Langsam geht es auch auf den anderen Baustellen voran. Etwa dort, wo nach osteuropäischem Vorbild neue Wohnblocks aus Betonfertigteilen entstehen - mit Stromanschluß, fließendem Wasser und Zentralheizung, aber keine architektonischen Kleinode.

Bei konzessiv zu interpretierendem aber bewirkt Konnektvertauschung klare Bedeutungsveränderung.

(9a) Das Haus hat ein kaputtes Dach, aber wir haben es gekauft.

(9b) Wir haben das Haus gekauft, aber es hat ein kaputtes Dach.

In (9a) ist eine konzessive Interpretation mit dem Alltagswissen kompatibel: Aber (in der Bedeutung, dennoch") signalisiert, dass der Sachverhalt des Hauskaufs entgegen der Erwartung eines Kaufverzichts infolge der Schadhaftigkeit des Dachs erfolgt. Diese Interpretation legt nahe, dass dem Sprecher der Zusammenhang präsent ist und folglich die mit dem Subjekt bezeichneten Personen um das schadhafte Dach wussten. In (9b) wird ein solches Wissen nicht vorausgesetzt und eine konzessive Interpretation scheint abwegig. (vgl. auch die Beispielpaare (1) bis (3) in Di Meola in diesem Band). Eine Kontrastinterpretation ist hier nach dem Muster von (8) als „Bewertungsgegensatz“ mit Hilfe eines Zwischenschritts möglich, indem man nämlich die nahe liegende Folgerung aus p nicht als direkte Negation von q sondern als Negation einer Folgerung $\mathrm{r}$ aus $\mathrm{q}$ versteht. Diese Zwischenschritte $\mathrm{r}$ und $\neg \mathrm{r}$ sind in (9b) eben die aus $\mathrm{p}$ und $\mathrm{q}$ folgerbaren konträren Sachverhaltsbewertungen durch den Sprecher: positiv - negativ.

Bei monosem konzessiven Konnektoren liegt immer eine asymmetrische Relation vor und eine Vertauschung der Konnekte lässt sich anders als in (9b) mit aber nicht mehr durch eine nicht-konzessive Lesart erklären, sondern führt zu einem unsinnigen Ergebnis, das allenfalls über eine Zusatzannahme wie „der Käufer ist professioneller Dachdecker“ reparierbar ist. ${ }^{9}$

9 Aufgrund der Asymmetrie und der unterschiedlichen Argumentstruktur von aber und obwohl (p, obwohl q: q = Blockierte Bedingung, $\mathrm{p}=$ Folgekontrast; $\mathrm{p}$, aber q: q = Folgekontrast) halte ich (10b) und (9a) für kein geeignetes Minimalpaar für die Ermittlung eines Bedeutungsunterschieds zwischen aber und obwohl; vgl. dagegen Di Meola (in diesem Band). 
(10a) Wir haben das Haus gekauft, obwohl es ein kaputtes Dach hat.

(10b) $\quad \neq$ Das Haus hat ein kaputtes Dach, obwohl wir es gekauft haben.

\subsection{Kontextabhängigkeit der Relationsbedeutungen}

Selbst bei der "symmetrischen Verwendung“ von und ohne ikonische Weiterinterpretation ergibt sich die Gemeinsame Einordnungsinstanz, unter der die Konnektbedeutungen zu bündeln sind, oft erst unter Berücksichtigung des weiteren Kontexts und Redehintergrunds und nicht unmittelbar aus den Konnektbedeutungen selbst. Dadurch werden selbst scheinbar inkohärente Verwendungen wie (11) (aus Lakoff 1971) reparierbar (nämlich unter der Annahme einer Wette des Subjektsreferenten, dass sechs Personen in einen Ford passen und er in diesem Fall seine Großmutter benachrichtigen würde).

(11) Sechs Personen passen in einen Ford und ich habe meiner Großmutter gestern einen Brief geschrieben.

Für aber und andere adversative Konnektoren zeigt sich wiederum ein differenzierteres Bild. Beim kontrastiven Vergleich ist der Kontrast durch sprachliche Indikatoren in den Konnekten verankert: durch kontrastierende Abschnitte in syntaktisch, semantisch und intonatorisch mehr oder minder parallel strukturierten Konnekten (zum Einfluss von Parallelität auf die Interpretation von aber vgl. Lang in diesem Band). Unmittelbar aus den Konnektbedeutungen ableitbar ist der Kontrast auch bei aber-Verknüpfungen wie (12), in denen das erste Konnekt eine Handlungsabsicht des Subjektsreferenten bezeichnet, - mitunter durch ein intensionales Prädikat wie wollen, versuchen, versprechen o.ä. lexikalisch direkt markiert - und das zweite Konnekt das Misslingen dieser Absicht; ein auch als , frustrated plan' bezeichneter Verwendungstyp von aber.

Warum der Staat von den Bürgern immer mehr Geld verlangt, aber immer weniger bekommt. (Spiegel-Titel vom 2.12.02)

Bei nicht-parallel strukturierten Konnekten ohne weitere lexikalische Indikatoren muss dagegen oft ein weiterer Kontext bemüht werden:

(13) Die Zeil bekommt ein ganz undeutsches Flair von Hydepark und italienischem Marktplatz. Aber Politik bleibt Männersache. (taz, 27.09.1986, S. 5)

(13) bleibt ohne Kontext ähnlich kryptisch wie (11). Hintergrund ist ein Unterrichtsprojekt, in dem Schüler auf der Frankfurter Zeil Passanten in Diskussionen zu brisanten Themen verwickeln. Der intendierte Kontrast zwischen ,poli- 
tisches Diskussionsforum für alle Bürger“ und „kein Diskussionsforum für Frauen“ wird erst aus der begründenden Textfortsetzung im Rückschluss ersichtlich:

[...] Die Hausfrauen schieben sich eilig vorbei und kümmern sich um den Einkauf.

Bei konzessiven Konnektoren bzw. bei konzessiver Interpretation von aber wird in starkem Maße auf konventionelle, kollektiv verfügbare Konzepte von Sachverhaltszusammenhängen zurückgegriffen. Aber selbst wenn diese dem Hörer nicht zur Verfügung stehen, braucht er für eine konzessive Interpretation nicht den weiteren sprachlichen Kontext. Vielmehr wird der Zusammenhang durch den konzessiven Konnektor selbst dann „mitgeliefert“, wenn, wie im folgenden, bei Umbach/Stede (1999: 5) und wieder in Stede (in diesem Band) diskutierten Beispiel, dem Hörer das Spezialwissen zum Nachvollziehen des präsupponierten Zusammenhangs fehlt.

$$
\text { Es war Juli, trotzdem haben wir keine Safranschirmlinge gefunden. }
$$

Fazit: Die in 3. auf der Basis von syntaktisch-strukturellen Parametern ermittelte skalare Anordnung von Additivität, Adversativität und Konzessivität spiegelt sich auch in den Dimensionen der semantischen Beschreibung. Mit der Zunahme der Grammatizität korreliert eine Abnahme in der semantischen Symmetrie und in der Kontext- und Situationsabhängigkeit. Umgekehrt erscheint der Kontrast zunehmend konzeptuell verankert. Dabei decken aberVerknüpfungen einen breiten Interpretationsraum ab, in dem ein Umschlag von der symmetrischen zur asymmetrischen Verknüpfung, von der kontextgebundenen zur konzeptgebundenen Interpretation stattfindet. Es dürfte deshalb lohnend sein, diesen Raum genauer unter die Lupe zu nehmen.

\section{5. aber: Grundbedeutung, Verwendungstypen und Verknüpfungsdomänen}

Die in 1. noch nicht exakt explizierte Beschreibung des Wesens von Kontrast soll nun präzisiert und für einige leicht typisierbare Verwendungskontexte ausbuchstabiert werden. Ein Sprecher, der p, aber q äußert, geht davon aus, dass - aus welchen Gründen auch immer - nicht q zur Debatte stand und dass der Hörer diese Annahme teilt. Formalisiert unter der Annahme strukturierter Propositionen wie in Pasch et al. (2003) gilt damit als Bedeutung von aber:

assertiert: $\quad \mathrm{p} \wedge \mathrm{q}$

diskurspräsupponiert: $\quad \neg q$ 
Da, wie in 4.3 gezeigt, für die Etablierung des Kontrasts unterschiedliche Quellen in Frage kommen (sprachliche Indikatoren im ersten Konnekt, weiterer Kontext, Schlussfolgerungen aus dem ersten Konnekt auf der Basis von Weltwissen), erfordert die Interpretation von aber-Verknüpfungen eine backtracking-Operation und ist eben auch dadurch komplexer als die von und-Verknüpfungen (Lang (2000: 247) spricht von einer „source-target-relation“ zwischen zweitem und einer Inferenz aus dem ersten Konnekt). Die hier gegebene Bedeutungsbeschreibung von aber deckt konzessive Interpretationen, bei denen $\neg q$ über das Konzept der „Normalerwartung“ sich unmittelbar als Implikation aus der Bedeutung des ersten Konnekts ergibt, mit ab, beschränkt sie aber nicht darauf, da sie die Quelle für die Präsupposition von $\neg q$ offen lässt. ${ }^{10}$

\section{$5.1 \quad$ Verwendungstypen von aber}

Insoweit aber speziellere Kontrastmarker vertreten kann, definiert seine Bedeutung auch die der allgemeineren Kontrastrelation. Innerhalb dieser Kontrastrelation lassen sich nun Verwendungstypen von aber herausschälen, in denen die Diskurspräsupposition auf je spezifische Weise zustande kommt und aber jeweils mit spezifischeren Kontrastkonnektoren austauschbar ist. ${ }^{11}$ Einige

10 Die obige Bedeutungsexplikation für aber geht zurück auf Pasch (1992). In den gängigen Beschreibungen in der Tradition von Anscombre/Ducrot (1977) ist nicht immer klar ersichtlich, inwieweit sie reine Beschreibungen von Konzessivität sind, oder inwieweit sie nichtkonzessive Verwendungen mit abdecken. Man vergleiche etwa König (1991): „Aber zeigt an, dass $\mathrm{p}$ und q im relevanten Kontext $\mathrm{K}$ (vor dem gegebenen Redehintergrund) zu einander widersprechenden Schlüssen $\mathbf{r}$ und $\sim \mathbf{r}$ berechtigen, wobei das zweite Konnekt und die darauf basierende Folgerung größeres argumentatives Gewicht hat"; Lötscher (1989: 218): „Eine Implikatur aus SA2 ist die Negation einer Implikatur aus SA1, wobei SA1 und SA2 die aus S1 und S2 im Kontext (c) sich ergebenden Annahmen sind“; Brauße (1983: 10): „direkte oder indirekte Negierung einer impliziten Schlussfolgerung“, „Zurückweisung einer unterstellbaren Folgerung“; Lang (2000: 13): „,indicating that the assertion rendered by the second clause is in contrast to an ASSUMPTION that either may be read off, or must be inferred from, previous information“. Auch bei Becker (1870) ist das bereits formuliert: „Zwei Gedanken stehen miteinander in einem adversativen Verhältnis, wenn der eine Gedanke nicht den anderen Gedanken selbst, sondern eine aus anderen Gedanken gezogene Folgerung aufhebt, in dem der Grund nur ein partieller, und die aus ihm gezogene Folgerung daher nicht eine notwendige sondern nur eine mögliche ist.“ (Becker 1870: 294)

11 Die jeweiligen „Äquivalente“ von aber werden hier weniger gründlich als bei Stede (in diesem Band) und - unter Absehung von Argumentstruktur und Stellungsrestriktionen vorläufig nur auf der Basis des eigenen Kompetenzurteils ermittelt; sie bedürften sorgfältigerer Untermauerung durch Belege. Auch ist das Verfahren nicht unproblematisch, worauf auch Stede hinweist, wenn (wie hier geschehen) davon abgesehen wird, dass adverbiale Konnektoren in Abhängigkeit von ihrer Position im Trägerkonnekt unterschiedliche Werte annehmen können. „Äquivalent“ bzw. „semantisch typgleich“ ist hier also als semantisch gleichwertig zu verstehen, und schließt Umstellungen in den Konnekten nicht aus. 
markante Verwendungstypen sollen hier isoliert und ihre „Trennschärfe“ an Belegen aus den Mannheimer Korpora überprüft werden.

\section{schwach kontrastives aber: Topicwechsel-Marker}

Der durch aber signalisierte Kontrast kann sich auf die Markierung eines Topicwechsels beschränken. In (15) und (16) werden nicht Konnektbedeutungen direkt oder indirekt kontrastiert, - denn dass ein Sprecher fragt und ein anderer antwortet, ergibt weder im Alltagswissen noch vor dem konkreten Texthintergrund einen Kontrast -, sondern andere Perspektivierungen und thematische Neuorientierungen in der Textfortsetzung markiert. Blatz (1970: 703) beschreibt diese Funktion sehr treffend: Das zweite Konnekt dient „zur Anknüpfung von etwas Neuem, das zwar als etwas von dem bereits erwähnten Verschiedenes, aber doch damit Zusammenhängendes aufgefaßt wird, wobei durch die Adversativpartikel oft nur die Überleitung zum folgenden und die Weiterführung der Rede bezweckt wird.“

Ich glaubte, nicht richtig verstanden $z u$ haben und machte ein etwas erstauntes Gesicht. ,Wohin?', fragte ich. Er aber, in humoristischdozierender Weise, antwortete: ,Ja, in die Synagoge der Israeliten. Es ist doch heute Freitag abend. ' (taz, 19.11.1990, S. 17-18)

Sobald der Knabe hinausgejagt war, erschien Jungfer Eli und ward in die Davert verbannt. Die Davert ist aber ein Wald im Münsterschen, wo Geister umgehen und wohin alle Gespenster verwiesen werden. (GRI/SAG, S. 94)

Topicwechsel ist nicht notwendig mit Topickontrastierung verbunden. In (16) lassen sich die fraglichen Topics, Jungfer Eli und die Davert, überhaupt nicht einer gemeinsamen Gegenstandsklasse zuordnen. Die Präsuppositionsbasis liegt bei diesem Typ in der allgemeinen Erwartung von Textkohärenz: Unterbrochen wird mit aber q die Unterstellung eines durchlaufenden Themas.

Topicwechsel markierendes aber findet sich vor allem in der schwachtonigen Nacherststellung unmittelbar hinter dem intonatorisch markierten Topic. Es ist eher in älteren narrativen Texten anzutreffen, rekurrent etwa in der Lutherbibel. Dort ist es eine wörtliche Übersetzung von altgr. de und lat. autem oder vero, die als DS-marker (für different-subject) fungieren, eine Markierung, die typischerweise in Pro-Drop-Sprachen entwickelt wird, in denen identisches Subjekt in einem Nachfolgesatz wegfallen kann. ${ }^{12}$

12 Im Übersetzungsvergleich zeigt sich, dass in vielen Sprachen (ebenso in neueren Übersetzungen) der adversative Konnektor nicht explizit wiedergegeben wird; (man vergleiche etwa bei http://www.diebibel.de/). 
(17) Und er sah sich um nach der, die das getan hatte. Das Weib aber fürchtete sich und zitterte, denn sie wußte, was an ihr geschehen war; sie kam und fiel vor ihm nieder und sagte ihm die ganze Wahrheit. Er aber sprach zu ihr: Meine Tochter, dein Glaube hat dich gesund gemacht; geh hin mit Frieden und sei gesund von deiner Plage!

(Markus 5, 25-34)

Semantisch typgleich: jedoch, wiederum, indessen, ?hingegen, ?dagegen (Blatz nennt außer aber noch dagegen, hingegen, hinwiederum in dieser Funktion.)

\section{kontrastiver Vergleich}

Beim kontrastiven Vergleich müssen die Topics kontrastieren; Voraussetzung dafür ist, dass sie vom selben semantischen Typ sind. Die akzentuelle Markierung weist sie als einander ergänzende Mitglieder einer im Diskurs zur Debatte stehenden geschlossenen Klasse aus. Gleichzeitig sind sie, so wie an Beispiel (1) demonstriert, bezüglich ihrer jeweiligen Charakterisierungen einander ausschließende Alternativen.
Beide Eltern sprachen „züritütsch“. Er aber habe auf keinen Fall ein "Zörihegel» sein wollen, sondern im Kindergarten ,blitzartig“ den Appenzellerdialekt gelernt. (St. Galler Tagblatt, 29.08.1998)

Die Akzentstruktur muss bei diesem Typ parallel sein; für die syntaktische Struktur gilt das nicht zwingend. Auch müssen die kontrastierten Topics keineswegs immer Subjekte sein (wie etwa bei Eggs (1977: 147) postuliert). Man vergleiche die zunehmend weniger parallelen Abwandlungen von (19).

(19a) SCHWEInebraten schmort man gut in DUNkelbier, aber RINderbraten besser in ROTwein.

(19b) SCHWEInebraten schmort man gut in DUNkelbier, aber bei RINderbraten macht sich ROTwein besser.

(19c) SCHWEInebraten schmort man gut in DUNkelbier, will man aber einen schmackhaften RINderbraten haben, ist ROTwein angesagt.

(20) HESsen, THÜringen und Teile von BAyern blieben ANgelsächsische Domänen, und LUL wurde Bischof von MAINZ, aber die KIRCHlichen Befehlsstellen wurden mit FRANken besetzt. (MK1/WPE)

Die dem kontrastiven Vergleich zugrunde liegende Präsupposition leitet sich aus der allgemeinen Erwartung her, dass auf typgleiche Entitäten gleiche Charakterisierungen zutreffen. Mit aber q wird diese Erwartung überschrieben. 
Semantisch typgleich: während, da(hin)gegen, dementgegen, demgegenüber, hingegen, jedoch, doch

Bei Typ (i) und (ii) liegen starke sprachliche (syntaktische und intonatorische, bei (ii) evtl. via Antonymie zusätzlich lexikalische) Indikatoren vor, die dem Hörer die Etablierung des Kontrasts direkt aus der Konnektbedeutung ermöglichen; ein expliziter interpretatorischer Zwischenschritt über Folgerungen aus den Konnektbedeutungen ist nicht nötig.

\section{(iii) Kontrast durch „Plan-Vereitelung“}

Charakteristisch für diese aber-Verwendungen ist, dass das erste Konnekt eine zielgerichtete Handlung des Sprechers oder des Subjektreferenten bezeichnet, und im zweiten Konnekt das Erreichen dieses Ziels negiert wird. Neben „frustrated plan" (Stede in diesem Band) findet sich dafür auch die Bezeichnung ,intentionales Widerspruchs-aber“ (Posch/Rieser 1976). Sprachliche Indikatoren dafür können intensionale Prädikate (wollen, beabsichtigen, versuchen o.ä.) im ersten Konnekt und Negationselemente (explizit in (21), lexikalisch in (22)) oder lexikalische Substitution wie in (23) oder (12) im zweiten Konnekt sein.

(21) Ein Gefangener der JVA Werl wollte sich Kukident kaufen. Das stand aber nicht auf der Liste der zugelassenen Kosmetika oder Chemikalien. (taz 12.02.1991, S. 22)

(22) Nach dreijähriger gemeinsamer Arbeit gedachte Willibrord, mittlerweile ein Mann in den Sechzigern, einen Teil der Verantwortung an den jüngeren Gefährten abzugeben. Bonifatius verzichtete aber auf die angebotenen Titel und Ehren. (MK1/WPE, S. 227)

(23) Die Chinesin wollte eigentlich Turnerin werden, aber Funktionäre entschieden, daß Wasserspringen für sie die geeignete Sportart wäre. (taz, 05.01.1991, S. 28)

Die Präsupposition $\urcorner q$ beruht hier auf dem naiven Schluss von der Absicht zu deren Verwirklichung. Das funktioniert ähnlich wie bei der skalaren Implikatur bei Ausdrücken wie viele, manche etc., wo aufgrund der Maxime der Quantität die alltagssprachliche Lesart enger ist, nämlich ,nicht alle', als die logische. Nach den Gesetzen der Logik präsupponieren intensionale Kontexte gerade das Nicht-Zutreffen des bezeichneten Sachverhalts und das aber in Er versuchte es, schaffte es aber nicht. wäre somit nicht gerechtfertigt, weil nicht im Widerspruch zur Schlussfolgerung aus p. In der alltagssprachlichen Interpretation intensionaler Kontexte ist jedoch offensichtlich die Planverwirkli- 
chung nicht ausgeschlossen, aber eben auch nicht zwingend, wie das scheinbar paradoxe Beispielpaar (24a, b) zeigt.

(24a) Fritz mogelte bei der Prüfung, aber er flog auf.

(24b) Fritz mogelte bei der Prüfung, aber er flog nicht auf.

Ersetzung durch monoseme konzessive Konnektoren (trotzdem, dennoch, obwohl) ist in Kontexten wie oben nicht möglich (?? Obwohl er es versuchte, schaffte er es nicht.); tatsächlich liegt hier auch nicht die konzessivitätstypische konditionale Beziehung des ,(normalerweise) wenn p, dann nicht q‘ zugrunde, sondern eher eine finale ,p, damit nicht q'. Eine Verschiebung zur Konditionalbeziehung und zur Konzessivinterpretation ist etwa durch Einfügung eines Modaladverbials möglich (Obwohl er es mit aller Kraft versuchte, schaffte er es nicht). Auch wenn die „Absicht“ nicht über einen intensionalen Ausdruck, sondern nur im Rückschluss aus dem Kontrast zum zweiten Konnekt erschließbar ist, ist eine Konzessivinterpretation möglich.

(25) Sie rüttelte ihn und rief ihn, aber sie konnte ihn nicht aufwecken. (= dennoch konnte sie ihn nicht aufwecken.) (GRI/KHM, S. 472)

(26) Der Vater aber sprach: „Kind, das ist kein Spielzeug, da hast du was Schönes angestiftet! Geh nur gleich und trag's wieder hinab ins Tal." Das Fräulein weinte, es half aber nichts. (= dennoch half es nichts.) (GRI/SAG, S. 47)

Semantisch typgleich: jedoch, doch, allerdings, freilich

(iv) Kontrast durch gegensätzliche Konnektbewertungen: „Bewertungsgegensatz-aber“

(27) Die schwarzen Massaibuben in der Serengeti machen sich einen Spaß daraus. Sie schleichen sich an ein schlafendes Nashorn heran und legen ihm einen Stein auf den Rücken. Der nächste muß dann wieder hin, um den Stein herunterzunehmen; das geht so fort, bis das Nashorn endlich aufwacht. Natürlich ist es kein ungefährliches Spiel, aber es paßt so richtig zu den Massai. (MK1/WGS, S. 52)

(28) Bis unser „Zebra" sich wieder wird in die Lüfte schwingen können, vergehen mindestens drei Wochen. Wir schleichen mit gestutzten Schwingen auf der Erde umher und kommen uns furchtbar langsam und unbeholfen vor. Aber wir haben jetzt Zeit, uns die sagenhafte Ritterburg näher zu besehen. (MK1/WGS, S. 91) 
(29) Deswegen ist es unerträglich, wenn heute noch ein indischer Minister in irgendeinem Lande als Farbiger im Hotel kein Unterkommen findet, während der ungebildete, aber europäische Farmer unrasiert gern aufgenommen wird. (MK1/WGS, S. 176)

Beim sogenannten „Bewertungsgegensatz-aber“ (Posch/Rieser 1976; andere Bezeichnung: „evaluativ“ (Eggs 1977)) ergibt sich der Kontrast als Gegenüberstellung eines positiven und eines negativen Werts, der den Konnektbedeutungen aus der Sicht des Sprechers zuzuschreiben ist (in (29) mit temporärer Übernahme einer Fremdperspektive durch den Sprecher). Auf der Ebene der Sachverhalte besteht in (27) und (28) weder ein lexikalischer Kontrast, noch eine konzessive Beziehung, da beide Konnekte Variationen ein und desselben Themas sind: ,gefährlich sein ' (in 27), ,Zeit haben“ in (28). Die Prädikate ungebildet und europäisch in (29) kontrastieren ebenfalls nicht, allenfalls ließe sich unter der entsprechenden Perspektive eine konzessive Beziehung (wenn jemand ungebildet ist, handelt es sich normalerweise um keinen Europäer) hypostasieren. Hinsichtlich der Werteverteilung positiv - negativ ist aber neutral, jedoch bestimmt der Wert des zweiten Konnekts die argumentative Richtung: In (29) passt die Anordnung negativer vor positivem Wert zu dem im Trägerkonnekt ausgedrückten Sachverhalt (man vgl. auch das analog konstruierte (8a)), und in (30) ergibt sich aus der Textfortsetzung, dass der quantitativ niedrigere Wert von $50 \mathrm{~km} / \mathrm{st}$ hier als der positive $\mathrm{zu}$ werten ist.

(30) Für gewöhnlich schnurrt es mit $220 \mathrm{~km} / \mathrm{st}$ durch die Lüfte. Aber wenn man die Landeklappen ausfährt, kann man bis auf $50 \mathrm{~km} / \mathrm{st}$ heruntergehen. Deswegen können wir auf Kartoffeläckern landen, wo man mit dem Auto nicht mehr fortkommt. (MK1/WGS, S. 24)

Semantisch typgleich: Kontrastkonnektoren, die bewertete Sachverhalte verknüpfen können, sind, wie in 4.2 dargelegt, teilweise nach der Zuordnung von Wert und Konnekt differenziert, worauf hier nicht weiter eingegangen wird. Auch weisen nicht alle Kontrastkonnektoren dieses Typs ihrem internen Konnekt stärkeres argumentatives Gewicht zu: So bezieht beispielsweise allerdings seine mehrfach beobachtete ,ausgleichende Funktion“ (Brauße 1983: 33) gerade aus der argumentativen Neutralität (vgl. Breindl 2003). Grundsätzlich kommen als Konnektoren in Kontrastkonstruktionen vom Typ Bewertungsgegensatz vor: jedoch, dafür, freilich, allerdings, einerseits (...) ander(er)seits, zum einen (...) zum anderen, immerhin, nur. 
Entscheidend für eine Konzessivinterpretation von aber (in der Bedeutung von trotzdem) ist die Rekonstruierbarkeit einer Implikation auf der präsuppositionalen Ebene, also eine Bedeutungsstruktur von assertiertem $\mathrm{p} \wedge \mathrm{q}$ und diskurspräsupponiertem p' $\rightarrow \neg q^{\prime}$ (mit p' und q' als geeigneten Generalisierungen über die singulären Sachverhalte $\mathrm{p}$ und $\mathrm{q}$ ). Bei monosemen konzessiven Konnektoren wird dieser Interpretationsprozess durch die Bedeutung des Konnektors ausgelöst und funktioniert, wie an (14) demonstriert, auch dann, wenn dem Hörer das Wissen um den Zusammenhang nicht zur Verfügung steht. Bei aber-Verknüpfungen wird der Interpretationsprozess komplizierter, denn die konzessive Interpretation ergibt sich via Implikatur als kausale Bedeutungsanreicherung des präsupponierten $\mathrm{p} \wedge \neg \mathrm{q}$ zu $\mathrm{p} \rightarrow \neg \mathrm{q}$ (vgl. Umbach/Stede 1999 und Breindl im Druck a). De facto geschieht dies oft erst durch Ausfiltern der anderen, kognitiv einfacheren, weil stärker sprachlich gestützten aberInterpretationen: In der aber-Version von (14) scheidet Kontrastvergleich wegen geringer Parallelität der Konnekte aus und Bewertungsgegensatz ist mit der Bedeutung des ersten Konnekts kaum vereinbar, Ersetzung von aber durch allerdings oder dafür ist nicht möglich. ${ }^{13}$

(14a) Es war Juli, aber wir haben keine Safranschirmlinge gefunden.

Das Konzessivschema des ,Widerspruchs gegen die Normalerwartung' darf freilich nicht als Kontrast zu einer logisch zwingenden, auf objektiven Zusammenhängen beruhenden generischen Konditionalbeziehung im Sinne eines ,immer wenn p, dann nicht q“ verstanden werden. So findet sich das in der Konzessivitätsliteratur vielstrapazierte short but strong, das oft in etwa so erklärt wird: „use of the word short triggers the expectation of weak, which is then denied by stating that John is strong." (Foolen 1991: 83), natürlich auch mit ganz anderen Kontrasten belegt.

(31) Prince ist kollegial und arbeitsam, schwarz und weiß und [...] klein, aber reich. (taz, 02.06.1992, S. 5)

(32) Er ist zwar klein, aber stark und lebenslustig. (taz, 15.10.1994, S.11)

(33) Chinesen [...] sind klein, aber zäh. (taz, 26.05.1998, S. 20)

(34) Der Bub ist klein, aber vif. (St. Galler Tagblatt, 13.07.1998)

13 Bewertungsgegensatz-Interpretation ist natürlich nicht gänzlich ausgeschlossen, verlangt aber ohne weitere sprachliche Indikatoren für das Vorliegen bewerteter Konnektbedeutungen erhebliche Zusatzannahmen. Entsprechende Abänderung des Beispiels macht sie plausibler:

(14b) Es war ein drückend heißer Julinachmittag. Aber wir haben Safranschirmlinge gefunden! / Dafür haben wir Safranschirmlinge gefunden! 
(35) Dann kommt eine Frau, so um die 50, klein, aber gewichtig, Sonnenbrille, langes Haar. (Frankfurter Rundschau, 11.7.1997, S. 21) Sie heißt Klein, und sie ist klein, aber sie hat eine sehr große und großartige Stimme. (taz, 26.03.1996)

Jetzt sitzen sie, klein, aber erwachsen, an der Seite der Sozialdemokraten. (Die Zeit, 01.10.1998, Nr. 41)

Die etablierten Kontraste lassen sich bei aller Verschiedenheit als Ausprägungen von ,effizient', ,gewichtig', ,ernst zu nehmen“ zusammenfassen - volkstümlich klein aber oho. Wieder andere Kontrastierungen finden sich bei Prädikationen über bewohnte Räume (klein, aber sauber/gemütlich/bezahlbar/originell) über Organisationen (klein, aber lautstark/effizient/angesehen/zukunftsweisend/mutig/selbständig) o.a. Der jeweils relevante Aspekt aus der Bedeutung des ersten Konnekts, zu dem im zweiten Konnekt ein Kontrast präsentiert wird, ergibt sich also zweifelsfrei erst im Rückschluss. Konzessivität als „Triggern“ oder „Auslösen“ einer (Normal-)Erwartung ist somit zu eng gegriffen. In einem konzeptuellen Schema scheint Kleinheit auf der Ebene der Sachverhalte relativ unspezifisch etwas wie geringe Effizienz zu evozieren; gleichzeitig ist Kleinheit auf einer Ebene der Bewertungen und Einstellungen mit einem niedrigen Wert korreliert. Tatsächlich lassen sich die obigen Beispiele auch als Kontrastierungen von Bewertungen lesen; aber entspricht dann nicht trotzdem, sondern dafür.

Für die Kodierung ist die Markierung einer Relation durch einen monosemen konzessiven Konnektor zunächst eine Entscheidung des Sprechers, die sicherlich durch konzeptuelle und alltagskulturelle Verankerung der Implikationsbeziehung begünstigt wird. Je stärker dieser Zusammenhang aus der Sicht des Sprechers ist - am stärksten natürlich bei naturgesetzlichen „ursächlichen“ Zusammenhängen -, desto eher wird er eine kontrastive Verknüpfung mit einem monosemen konzessiven Konnektor markieren.

\begin{abstract}
Das Lesen ging ihm glatt von der Zunge, und Latein beherrschte er wie seine Muttersprache. Aber schon mit dem Griechischen hatte er seine Not. Schreiben hat er nie richtig gelernt, obwohl er, wie der getreue Einhard berichtet, stets ein Schreibtäfelchen unter dem Kopfpolster seines Bettes hatte. (MK1/WPE, S. 285)
\end{abstract}

In (38) folgt offenbar aus der Kenntnis des Lateinischen für den Schreiber nicht so zwingend die Kenntnis des Griechischen, wie aus dem Besitz einer Schreibtafel die Beherrschung des Schreibens folgt. ${ }^{14}$ Sachlogisch ist jedoch

14 Ein Blick in die Mannheimer Korpora scheint eine solche Kodierungsstrategie zu bestätigten: Bei einer Durchsicht von 50 per Zufallsgenerator ermittelten aber-Verwendungen erwies sich der Anteil der eindeutig konzessiven Verwendungen, die sich ohne Bedeutungsverschiebung 
keine der beiden Schlussfolgerungen zwingend, sodass eine Vertauschung für den Hörer hier vermutlich nicht weiter auffällig wäre. Konzessivität basiert also nicht auf einer ontologischen Ebene der in der Welt vorzufindenden Implikationen, sondern auf der Ebene von „Annahmen über Kookkurrenzen von Sachverhalten“" (Dufter 2003: 60).

Semantisch typgleich: jedoch, dennoch, nichtsdestotrotz, trotzdem, dennoch, obwohl, obgleich, obzwar, obschon, wenngleich

\subsection{Verknüpfungsdomänen von aber}

Eve Sweetser (1990) führt unter den ,pragmatisch ambigen“ Satzverknüpfern, die außer auf einer Sachverhaltsebene (,content domain“) auch auf einer „epistemischen" Ebene der Annahmen, Urteile und Bewertungen sowie auf einer Ebene von Sprechakten verknüpfen können, auch but an (ebd. 100ff.). Das mag für kausale Konnektoren nachvollziehbar sein, wie die lebhafte SweetserRezeption seither gezeigt hat (zu den Verknüpfungsebenen bei bevor und nachdem s. Blühdorn in diesem Band); ihre Analysen von but-Verknüpfungen und ihre Hauptthese, eine „content-domain“ Lesart sei hier nicht zweifelsfrei nachweisbar, lässt jedoch einige Fragen offen, insbesondere die nach dem Verhältnis dieser Ebenen zu den traditionell unterschiedenen und den hier in 5.1 identifizierten Verwendungstypen von but resp. aber. So veranschlagt Sweetser, wie Lang (2000) gezeigt hat, die Rolle formaler Indikatoren (Topologie, Intonation, Grad der Parallelität der Konnektstruktur) und die Möglichkeit ebenenspezifischer Distribution der einzelnen Konnektoren zu gering, demzufolge sei keineswegs jede konnektorale Verknüpfung prinzipiell mehrfach ,pragmatisch ambig“. Unstrittig ist eine sprechaktverknüpfende Funktion von aber. Dabei kontrastiert der durch das zweite Konnekt repräsentierte Sprechakttyp mit einem Sprechakt, auf dessen Vollzug man aufgrund der ÄuBerung des ersten Konnekts schließen könnte. Formale Anzeichen dafür sind Verschiedenheit des Satzmodustyps und separate Intonationskonturen der Konnekte, wie in (39) (= Langs (3b)), wo die mit dem ersten Konnekt ausgedrückte Einräumung eher eine Fortsetzung mit einer expliziten Entschuldigung erwarten lässt.

(39) Wir sind mit dem Manuskript in Verzug, aber welcher Autor hält schon Termine ein?

durch eine $o b w o h l$-Verknüpfung ersetzen ließen, als eher gering; er liegt in jedem Fall unter 10 Prozent. 
Die von Sweetser als „epistemisch“ klassifizierten aber-Verknüpfungen Fälle von kontrastivem Vergleich und konzessivem aber - weist Lang zurück; sie seien als Verknüpfungen von Sachverhalten (Statements) interpretierbar, deren „epistemischer“ Anteil in der grundsätzlichen Beteiligung von Inferenzen aus dem ersten Konnekt bei der Interpretation von aber-Verknüpfungen liege. Dieser Anteil gehört jedoch zur semantischen Grundbedeutung von aber und ist nicht aufhebbar. Gegen Sweetser, die diese beiden Konzepte vermischt, sei aber zu unterscheiden zwischen ,inference (whether a proposition is asserted or inferred) and epistemic status (whether a proposition forms the content of a factual STATEMENT or an ASSUMPTION)“ (Lang 2000: 12). Tatsächlich ist Sweetsers Interpretation einer „real-world“-Lesart von but überhaupt nicht mit dessen Grundbedeutung verträglich: Sie schließt eine solche Lesart von John is rich but Bill is poor. mit der Begründung aus, ,there is no bar in the real world to the simultaneous existence of poor and rich people" (ebd. 103). Genau diese simultane Existenz von $\mathrm{p}$ und $\mathrm{q}$ ist aber Bestandteil der Grundbedeutung von aber resp. but - genauso wie von und resp. and, welch letzteres nach Sweetser in allen drei Domänen verknüpfen kann.

Typ (iv), das Bewertungsgegensatz-aber, wird von Sweetser wiederum den Sprechaktverknüpfungen zugeschlagen: hier stünden nicht Annahmen des Sprechers zueinander im Kontrast, sondern ,the speaker seems to be simultaneously proposing two mutually exclusive options" (ebd. 101f.). Aber auch bei diesem Typ werden Inferenzen aus den Konnekten kontrastiert, nämlich Bewertungen im Rahmen einer Argumentation, nicht aber Sprechakte und Sprechakttypen wie in (39). Überdies ist der zu inferierende Kontrast ja gerade nicht propositional ausgedrückt und erlaubt deshalb oft mehr als eine Rekonstruktion. Und insofern Bewertungen immer auch mit kulturspezifischen, in einer Sprechergemeinschaft geteilten Werten zusammenhängen, sind sie auch Bestandteil des Alltagswissens. So lässt sich etwa in (29) der Kontrast ungebildet, aber europäisch auf einen sprechersubjektiven Bewertungsgegensatz negativ - positiv zurückführen; (dann ist aber austauschbar mit dafür oder freilich); möglich ist aber ebenso eine Rekonstruktion nach dem konzessiven Muster unter Bezug auf eine in der hier hypostasierten Wertegemeinschaft geteilte Annahme, dass ,Europäer sein' und ,ungebildet sein “ miteinander unverträgliche Tatbestände sind: Bei dieser Interpretation ist aber äquivalent mit obwohl, obgleich etc. Und auch in vielen Fällen, in denen aber nicht durch obwohl ersetzbar ist, bleibt unklar, ob der Kontrast unter Bezug auf eine sprechersubjektive Bewertung oder unter Bezug auf allgemein geteiltes Alltagswissen herzustellen ist. So etwa in (41), wo neben dem subjektiven Bewertungskontrast auch ein objektiver Zusammenhang unterstellt werden kann, zwischen schwacher Blutung und geringer Gefahr, die zu der aus der Tiefe der Wunde abzuleitenden Gefährlichkeit im Widerspruch steht. 

zu sein. (MK1/WGS, S. 153)

Eine Subklassifikation der aber-Verknüpfungen - bei denen ja allein aufgrund der Bedeutung von aber immer Diskurspräsuppositionen und Inferenzen von Sprecher und Hörer beteiligt sind -, entlang der Sweetserschen Domänen scheint mir deshalb im Vergleich zu einer Klassifikation nach dem Typ und der Quelle der zum zweiten Konnekt in Kontrast stehenden Inferenz von geringerer Distinktionskraft.

\section{Das interpretatorische Schillern von aber}

Die hier angeführten Typen sind an idealtypischen konstruierten Beispielen und kontextfrei präsentierten Belegen exemplifiziert und differenziert worden. Nicht selten lassen aber-Verknüpfungen jedoch mehr als eine Herleitung des Kontrasts zu und sind dann nicht eindeutig auf einen Typ festzulegen. Der Ersetzungstest ergibt in diesen Fällen kein klares Bild. Insbesondere ist der subjektive Faktor beim Bewertungsgegensatz nicht sauber zu trennen von der Konzessivität (vgl. 5.3). Auf die Affinität von Konzessivitätsinterpretation und skalarer Implikatur wurde schon hingewiesen (vgl. 5.2, Typ (iii)). Selbst der kontrastive Vergleich ist nicht immer eindeutig. Welche Inferenzen ausgelöst werden, bestimmt sich letztlich durch den Diskurskontext. So kann unser Eingangsbeispiel Die Eltern sprechen türkisch, aber die Kinder sprechen deutsch. unterschiedlich interpretiert werden und aber korreliert dann mit unterschiedlichen Mengen von Kontrastkonnektoren:

(2d) kontrastiver Vergleich (hingegen, dagegen, während):

[A: In der Familie wird wohl deutsch gesprochen? B: Teils, teils,] Die Eltern sprechen türkisch, aber die Kinder sprechen deutsch.

(2e) konzessiv (dennoch, trotzdem, obwohl) (vgl. auch das ambige (18)): [A: Konnten die Kayas ihren Kindern türkisch beibringen? B: Nein,] Die Eltern sprechen türkisch, aber die Kinder sprechen deutsch.

(2f) Bewertungsgegensatz positiver Pol (immerhin, dafür, allerdings): [A: Hat sich Familie Kaya gut integriert? B: Naja,] Die Eltern sprechen türkisch, aber die Kinder sprechen deutsch.

(2f') Bewertungsgegensatz negativer Pol (nur, allerdings):

[A: Konnten die Kayas ihre Traditionen bewahren? B: Naja,] Die Eltern sprechen türkisch, aber die Kinder sprechen deutsch.

Die idealtypischen Verwendungsmuster von aber sind Ergebnis einer Typisierung der Konnektstruktur, des Kontexts und des zur Interpretation bemühten Rekonstruktionsmusters. Sofern ein bestimmtes Muster mit einer spezifischen 
Menge von typgleichen Kontrastkonnektoren korreliert ist, ist die Annahme eines spezifischen Subtyps begründet. Dass es auch sinnvoll sein kann, aus anderen Beweggründen weitere Subtypen zu etablieren, sei unbestritten; sie basieren in aller Regel auf feinerer Typisierung der Kontexte. Dies erklärt zusammen mit dem interpretatorischen Schillern sowohl die auffällige Divergenz zwischen den 8 Kontrasttypen bei Stede (in diesem Band), den 6 abers bei Posch/Rieser (1976), den Lakoffschen 2 abers (Lakoff 1971), den 3 Typen bei Eggs (1977) und dem monosemen bei Foolen (1991) und anderen, als auch die Tatsache, dass mitunter der selbe Beispieltyp unterschiedlich klassifiziert wird. Insofern verstehen sich die hier genannten Typen als „leicht identifizierbare Nester" in der allgemeinen Kontrast-Bedeutung, und nicht als exhaustive Klassifikation. Die unterspezifizierte Bedeutung von aber lässt im Zusammenwirken mit dem Kontext spezifischere Intepretationen zu, kann aber eben auch, da die involvierten Schlüsse nicht verbalisiert werden, für den Hörer ambig sein.

\section{Quellen}

Die Belege entstammen großteils den Mannheimer Korpora für geschriebene Sprache. Öffentlich zugänglich unter: http://www.ids-mannheim.de/cosmas $2 /$

MK1/WGS: Bernhard Grzimek, Michael Grzimek, Serengeti darf nicht sterben. Ullstein, Berlin/Frankfurt M./Wien 1967.

MK1/WPE: Rudolf Pörtner, Die Erben Roms, Roman. Econ Verlag, Düsseldorf 1964.

GRI/SAG: Deutsche Sagen, gesammelt von Jacob und Wilhelm Grimm; Erstveröffentlichung 1816 und 1818 .

\section{Literatur}

Anscombre, Jean-Claude/Ducrot, Oswald (1977): Deux mais en français? In: Lingua 43, S. 23-40.

Becker, Karl Ferdinand (1969): Ausführliche deutsche Grammatik als Kommentar der Schulgrammatik. Hildesheim, New York: Olms (Reprografischer Nachdruck der Ausgabe Prag 1870).

Birkner, Karin/Dimroth, Christine/Dittmar, Norbert (1995): Der adversative Konnektor aber in den Lernvarietäten eines italienischen und zweier polnischer Lerner des Deutschen. In: Handwerker, Brigitte (Hg.): Fremde Sprache Deutsch. Tübingen: Narr. (Tübinger Beiträge zur Linguistik 409), S. $65-118$. 
Blatz, Friedrich (1970): Neuhochdeutsche Grammatik mit Berücksichtigung der historischen Entwickelung der deutschen Sprache. Hildesheim, New York: Olms.

Brauße, Ursula (1983): Bedeutung und Funktion einiger Konjunktionen und Konjunktionaladverbien: aber, nur, immerhin, allerdings, dafür, dagegen, jedoch. In: Lang, Ewald (Hg.): Untersuchungen zu Funktionswörtern (Adverbien, Konjunktionen, Partikeln). Berlin: Akademie der Wissenschaften der DDR. (Linguistische Studien des ZISW 104), S. 1-40.

Brauße, Ursula (1998): Was ist Adversativität?: aber oder und? In: Deutsche Sprache 26/2, S. 138-159.

Breindl, Eva (2003): Das Rätsel um das paradoxe allerdings: (K)ein Fall für die Textgrammatik der deutschen Sprache? In: Thurmair, Maria/Willkop, Eva-Maria (Hgg.): Am Anfang war der Text - 10 Jahre „Textgrammatik der deutschen Sprache“. München: iudicium, S. 73-94.

Breindl, Eva (im Druck a): Konzessivität und konzessive Konnektoren im Deutschen. In: Deutsche Sprache.

Breindl, Eva (im Druck b): Polysemie und Invarianz bei Konnektoren: allerdings und andere Kontrastmarker. In: Pohl, Inge/Konerding, Klaus Peter (Hgg.): Prozesse der Bedeutungskonstruktion. Frankfurt a. M.: Lang.

Di Meola, Claudio (1997): Grammatikalisierungsprozesse am Beispiel subordinativer Konzessivkonnektive. In: Papiere zur Linguistik 57, S. 183-203.

Dufter, Andreas (2003): Konzessivität als markierte Kookkurrenz. In: Blank, Andreas/Koch, Peter (Hgg.): Kognitive romanische Semasiologie und Onomasiologie. Tübingen: Niemeyer. (Linguistische Arbeiten 467), S. 5776.

Eggs, Eckehard (1977): Zum Verhältnis von Grammatik- und Wirklichkeitskenntnis in Konzessivsätzen (am Beispiel des Französischen). In: Papiere zur Linguistik 12, S. 116-158.

Foolen, Ad (1991): Polyfunctionality and the Semantics of Adversative Conjunctions. In: Multilingua 10, S. 79-92.

Heidolph, Karl-Erich et al. (1981): Grundzüge einer deutschen Grammatik. Berlin: Akademie-Verlag.

König, Ekkehard (1985): Where do Concessives come from? In: Fisiak, Jacek (Hg.): Historical semantics - historical word-formation. Berlin u.a.: Mouton de Gruyter. (Trends in Linguistics/Studies and Monographs 29), S. 263-282.

König, Ekkehard (1991): Konzessive Konjunktionen. In: Stechow, Arnim von/ Wunderlich, Dieter (Hgg.): Semantik. Ein internationales Handbuch der zeitgenössischen Forschung. Berlin/New York: de Gruyter. (HSK 6), S. 631-639. 
König, Ekkehard/Auwera, Johan van der (1988): Clause Integration in German and Dutch conditionals, concessive conditionals, and concessives. In: Haiman, John/Thompson, Sandra A. (Hgg.): Clause combining in grammar and discourse. Amsterdam, Philadelphia: Benjamins, S. 101-133.

Kortmann, Bernd (1998): Adverbial Subordinators in the languages of Europe. In: van der Auwera, Johan (Hg.): Adverbial Constructions in the languages of Europe. Berlin, New York: de Gruyter. (Empirical approaches to language typology 20), S. 457-561.

Lakoff, Robin (1971): If 's, and's, and but's about Conjunction. In: Fillmore, Charles J./Langendoen, D. Terence (Hgg.): Studies in Linguistic Semantics. New York u.a.: Holt, Rinehart and Winston, S. 114-149.

Lang, Ewald (1977): Semantik der koordinativen Verknüpfung. Berlin: Akademie-Verlag. (studia grammatica 14).

Lang, Ewald (1991): Koordinierende Konjunktionen. In: Stechow, Arnim von/ Wunderlich, Dieter (Hgg.): Semantik. Ein internationales Handbuch der zeitgenössischen Forschung. Berlin, New York: de Gruyter. (HSK 6), S. 597-623.

Lang, Ewald (2000): Adversative Connectors on Distinct Levels of Discourse: A Re-examination of Eve Sweetser's Three-level Approach. In: Kortmann, Bernd/Couper-Kuhlen, Elizabeth (Hgg.): Cognitive and Discourse Perspectives on Cause, Condition, Concession, and Contrast. Berlin, New York: de Gruyter, S. 235-256.

Lehmann, Christian (1995): Synsemantika. In: Jacobs, Joachim et al. (Hgg.): Syntax. Ein internationales Handbuch der zeitgenössischen Forschung. Berlin, New York: de Gruyter, S. 1251-1265.

Lehmann, Christian (1995): Thoughts on Grammaticalization. München, Newcastle: Lincom Europa.

Lötscher, Andreas (1989): Implikaturen und Textfunktion im Gebrauch von Konnektiven des Gegensatzes. In: Linguistische Berichte 121, S. 215-240.

Pasch, Renate (1992): Kausale, konzessive und adversative Konnektive: Konnektive als Mittel des Ausdrucks von Diskurspräsuppositionen. In: Beckmann, Susanne (Hg.): Semantik. Münster: LIT-Verlag. (Münstersches Logbuch zur Linguistik 1), S. 33-48.

Pasch, Renate/Brauße, Ursula/Breindl, Eva/Waßner, Ulrich Hermann (2003): Handbuch der deutschen Konnektoren. Linguistische Grundlagen der Beschreibung und syntaktische Merkmale der deutschen Satzverknüpfer (Konjunktionen, Satzadverbien und Partikeln). Berlin, New York: de Gruyter. (Schriften des Instituts für Deutsche Sprache 9).

Posch, Günter/Rieser, Hannes (1976): Adversative Satzverknüpfungen mit aber. Konstanz: Universität Konstanz. (Arbeitspapiere des SFB 99).

Posner, Roland (1979): Bedeutung und Gebrauch der Satzverknüpfer in den natürlichen Sprachen. In: Grewendorf, Günther (Hg.): Sprechakttheorie und Semantik. Frankfurt a. M.: Suhrkamp, S. 345-385. 
Rudolph, Elisabeth (1996): Contrast. Adversative and concessive relations and their expressions in English, German, Spanish, Portuguese on Sentence and Text Level. Berlin, New York: de Gruyter.

Sweetser, Eve E. (1990): From Etymology to Pragmatics. Metaphorical and Cultural Aspects of Semantic Structure. Cambridge u.a.: Cambridge University Press.

Umbach, Carla/Stede, Manfred (1999): Kohärenzrelationen: Ein Vergleich von Kontrast und Konzession. In: KIT Report 148 (FB Informatik, TU Berlin). http://flp.cs.tu-berlin.de/publikationen/kit/r148/Kohaerenzrelationen.pdf 